\title{
The HPM Live $\mu$ - From Live Cell Imaging to High Pressure Freezing in Less than 2 Seconds for Correlative Microscopy Approaches
}

\author{
Xavier Heiligenstein ${ }^{1,2,4}$, Martin Belle ${ }^{3,4}$, Frédéric Eyraud ${ }^{3}$, Graça Raposo ${ }^{1,2}$, Jean Salamero ${ }^{1,2}$, Jérôme \\ Heiligenstein $^{3}$ \\ ${ }^{1 .}$ Cell and Tissue Imaging Facility CNRS Institut Curie, PSL Research University, Paris, France \\ 2. CNRS UMR 144, Paris, France \\ 3. CryoCapCell, Paris, France
}

Correlative Light and Electron Microscopy (CLEM) approaches allow linking molecular function to ultrastructure. Although no longer considered as an emerging methodology, technological developments are still required to turn the method into routine. From sample preparation to image registration, several gaps remain to be filled before it turns into a beginner's approach.

For optimal preservation at electron microscopy level, and observe ultrastructure in close to native state, vitrification is to date considered the best technology. As vitrification can be achieved with several techniques, only one allows deep freezing with ultrastructure preservation down to 200um deep: High Pressure Freezing. This technique applies a high cooling rate (>10000K/s) under high pressure (2048 bars) to achieve deep vitrification. The approximately 2000 bars required impose to protect the sample from the liquid nitrogen jet. Keeping in mind the sample preparation as a critical step, we developed several years ago a transparent carrier designed for correlative light and electron microscopy using HPF: the CryoCapsule [1].

Around this technology breakthrough, we designed a new generation of High Pressure Freezing Machine, directly linked to a light microscope to vitrify a specimen in less than 2 seconds: The HPM Live $\mu$.

In combination with our latest image registration software [2], we can achieve high spatio-temporal resolution in CLEM approaches for cell biology [3]. 


\section{References:}

[1] X. Heiligenstein et al, Traffic 15(6) (2014), p. 700.

[2] X. Heiligenstein et al, Nat. Methods 14(2) (2017), p. 102.

[3] The authors thank the France-BioImaging infrastructure supported by the French National Research Agency (ANR-10-INSB-04, "Investments for the future"). X.H. was funded by the Labex grant No. ANR-10-LBX-0038 part of the IDEX Idex PSL No. ANR-10-IDEX-0001-02 PSL. We acknowledge the FRM grant No. DEQ20140329491. MB, FE and JH are members of the CryoCapCell company.

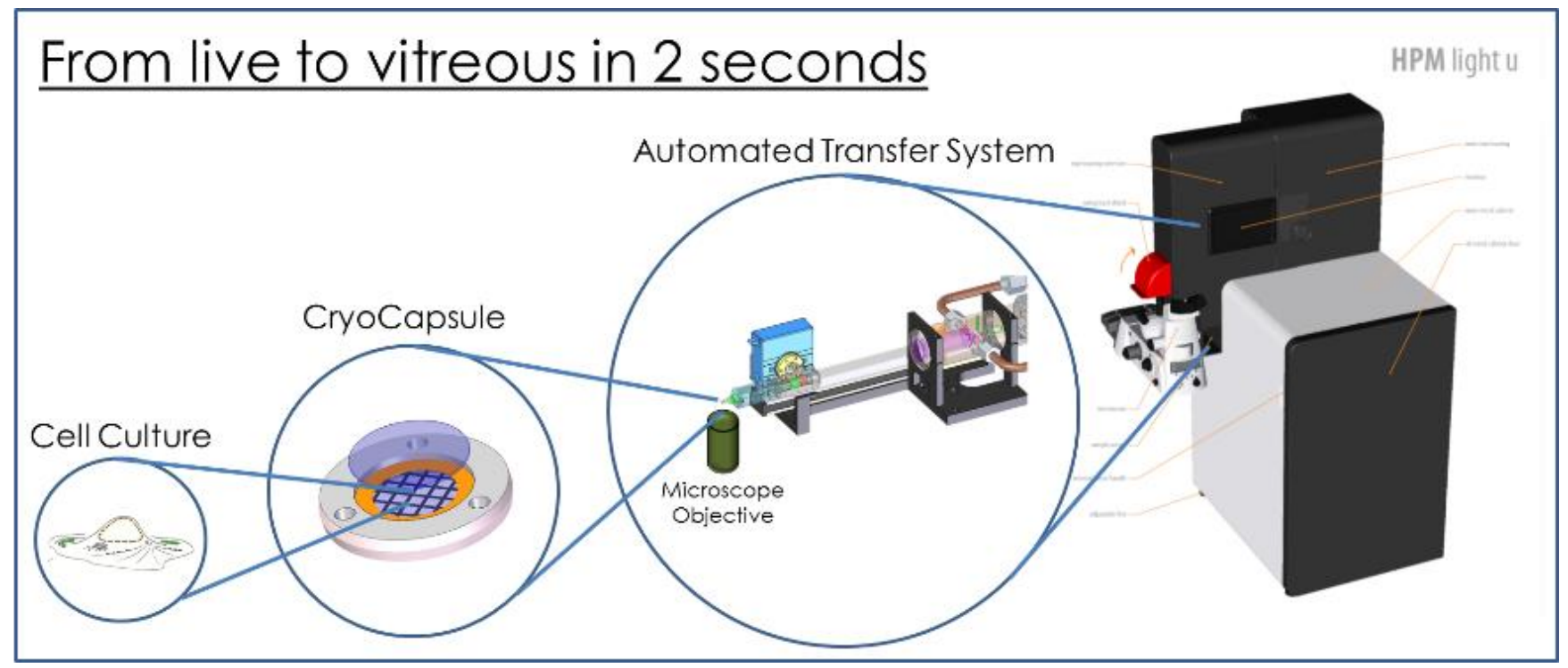

Figure 1. HPM live $\mu$ : A new generation of High Pressure Freezer, associated to a light microscope. The sample is loaded in the CryoCapsule before the experiment. The CryoCapsule is then loaded on top of the microscope objective where live cell imaging is performed. When commanded by the user, the sample is automatically transferred for vitrification in the HPM Live $\mu$. 